\title{
Verantwortlichkeiten der MRT-Anwendung in der Forschung
}

\section{Einführung \\ $\nabla$}

Die Durchführung von MRT-Untersuchungen bei klinischen und / oder wissenschaftlichen Untersuchungen kann hinsichtlich der strahlenschutzrechtlichen und arzthaftungsrechtlichen Vorgaben anderen Anforderungen unterliegen, als in der Regel- bzw. Krankenversorgung.

Drei grundsätzliche Untersuchungskategorien sind hierbei zu unterscheiden:

- die ausschließlich klinisch indizierte

Bildgebung (hier im Wesentlichen nicht in der Diskussion),

- Bildgebung bei kombinierter klinischer und wissenschaftlicher Fragestellung,

- ausschließlich wissenschaftlich veranlasste Bildgebung.

Für den Radiologen als Geräteverantwortlichen stellt sich dabei einerseits die Frage, ob auch bei einer rein aus Forschungsgründen durchgeführten Untersuchung stets ein fachkundiger Arzt anwesend sein muss. In diesem Zusammenhang ist zu klären, wie die Anforderungen an die Fachkunde auszulegen sind. In der Praxis wird teilweise angeführt, dass außeruniversitäre Organisationen wie z. B. das Forschungszentrum Jülich oder das Bender Institute of Neuroimaging im Fachbereich Psychologie und Sportwissenschaft der Universität Gießen für reine Forschungszwecke selbstständig MR-Systeme ohne fachkundige Ärzte oder Fachärzte betreiben.

Insoweit stellt sich auch die Frage, ob es rechtlich einen Unterschied darstellt, wenn man als Studienteilnehmer in einem Umfeld mit ärztlichen Mitarbeitern (wie auf dem Gelände eines Universitätsklinikums und einer Medizinischen Fakultät) untersucht wird oder in einem reinen Forschungsinstitut außerhalb der Krankenversorgung. Über diese Fragestellung hinausgehend ist zu hinterfragen, wie in rechtlicher Hinsicht bei rein wissenschaftlich indizierten Untersuchungen an Probanden bei einem unerwarteten Auftreten von Nebenbefunden in optimaler Weise verfahren werden sollte.

In der aktuellen Literatur werden derzeit 3 Wege bzgl. des Umgangs mit diesen Nebenbefunden diskutiert:
1. Der Proband wird offensiv darüber aufgeklärt, dass er ausschließlich aus Forschungsgründen untersucht wird und nicht über möglicherweise medizinisch für ihn relevante Befunde aufgeklärt wird. Wenn er dazu nicht bereit ist, kann er an der Studie nicht teilnehmen.

2. Der Proband wird darüber informiert, dass die rein aus Forschungsgründen aufgenommenen Sequenzen grundsätzlich durch einen fachkundigen Arzt, in diesem Fall durch einen Radiologen bzw. Neuro- oder Kinderradiologen, diagnostisch befundet werden, weist jedoch darauf hin, dass Forschungssequenzen in der Regel nur bedingt eine suffiziente Diagnostik ermöglichen und der Befund daher u.U. nur einen provisorischen Charakter hat und ggf. durch weiterführende Untersuchungen ergänzt werden sollte.

3. Proaktiv wird das rein wissenschaftliche Forschungsprotokoll zusätzlich mit Teilen einer klinischen Untersuchung ergänzt, sodass insgesamt neben der Erhebung von Forschungsdaten auch die Möglichkeit einer adäquaten diagnostischen Beurteilung gegeben ist, welche dann obligat durch einen fachkundigen Radiologen erfolgt. Diese Befunde werden dem Patienten anschließend obligat mitgeteilt. Wenn er dazu nicht bereit ist, kann er an der Studie nicht teilnehmen.

Soweit im Rahmen einer MRT-Messung aus Forschungsgründen, z.B. durch Psychologen oder Neurologen in der radiologischen Klinik eines Universitätsklinikums oder eines Krankenhauses bei einem Probanden Anomalien festgestellt werden, ist es daher fraglich, ob der Radiologe, in dessen Abteilung das MRT steht, verpflichtet ist, die Bilder mit Blick auf eine mögliche Erkrankung zu analysieren und ggfls. zu befunden. Gerade im Arzthaftungsrecht kommt es oft auf die Details im Einzelfall an; jedoch soll nachstehend versucht werden, durch eine generalisierende Betrachtung die möglichen Problemkreise aufzuzeigen.

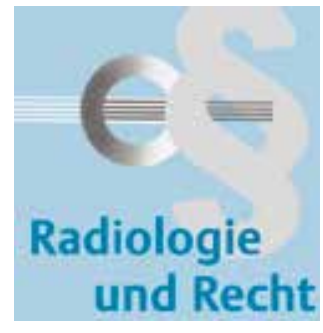

\section{Beachtung von Zufallsbefunden im Rahmen von Studien $\nabla$}

Vor einer Beurteilung der Frage nach der Haftung im Rahmen von Zufallsbefunden muss zunächst die grundsätzliche Anwendbarkeit der Regelungen des Arzthaftungsrechts festgestellt werden.

\section{Grundsätzliches}

Festzustellen ist, dass zum einen eine vertragliche Haftung des Arztes aus dem sog. Behandlungsvertrag existiert, soweit der behandelnde Arzt eine eigenständige vertragliche Beziehung mit dem Patienten eingeht (z.B. wahlärztliche Leistungen des liquidationsberechtigten Krankenhausarztes). Seit Erlass des Patientenrechtegesetzes vom 20.02.2013 ist der Behandlungsvertrag in den $\S \S 630$ a ff. BGB kodifiziert. Auch im Rahmen einer rein wissenschaftlichen Studie existiert zumindest ein Probandenvertrag, der ein Schuldverhältnis gemäß § 241 BGB mit hieraus resultierenden Pflichten darstellt. Dabei dürfte es nicht abwegig sein, die rechtlichen Wertungen des durchaus vergleichbaren Behandlungsvertrags für den Probandenvertrag heranzuziehen. Das Oberlandesgericht Köln hat die analoge Anwendbarkeit der Vorschriften des Behandlungsvertrags nach § 630a BGB auch auf andere Konstellationen im Gesundheitswesen (im konkreten Fall die Abgabe von Medikamenten in der Apotheke) ausgedehnt (OLG Köln, Urt. v. 07.08.2013, Az.: 5 U 92/12 = Arzneimittel \& Recht 6/2013, S. 296 ff. mit Anmerkung Wigge / Frigger).

Zum anderen wird eine gesetzliche Haftung aus unerlaubter Handlung nach den Vorschriften des Deliktsrechts gemäß der $\S \S 823 \mathrm{ff}$. BGB begründet, nach denen ein Arzt oder ein Nichtarzt haften, die vorsätzlich oder fahrlässig das Leben, den Körper oder die Gesundheit des Patienten widerrechtlich verletzen. Der Arzt ist dem Patienten dann zum Ersatz des daraus entstehenden Schadens verpflichtet. Diese deliktische Haftung ist unabhängig von 
einem vorliegenden Vertragsverhältnis immer zu beachten.

Dabei ist es festzuhalten, dass die Grundsätze des Arzthaftungsrechts auch im Bereich wissenschaftlicher Studien Anwendung finden (Lippert, in: Ratzel/Lippert, Kommentar zur Muster-Berufsordnung der deutschen Ärzte (MBO), § 15 MBO, 4. Auflage 2006, Rn. 48). Hinsichtlich der vertraglichen Haftung muss allerdings beachtet werden, dass diese sich nicht auf den konkreten Arzt, sondern auf die Einrichtung bezieht, mit der der Probandenvertrag geschlossen wird (vgl. Lippert a.a.O.).

Im Rahmen der Rechtsdogmatik des Arzthaftungsrechts könnte es sich bei einer Nichtabklärung der im Rahmen von Studien entdeckten möglichen Anomalien um sogenannte Diagnosefehler, aber auch um sogenannte Befunderhebungsfehler handeln.

Ein Diagnosefehler liegt dann vor, wenn der Arzt in irgendeiner Form vorliegende Befunde falsch interpretiert und deswegen nicht die aus der berufsfachlichen Sicht seines Fachbereichs gebotenen Maßnahmen ergreift. Ein Befunderhebungsfehler ist dagegen gegeben, wenn die $\mathrm{Er}$ hebung medizinisch gebotener Befunde unterlassen wird. Nach der Rechtsprechung liegt dann ein Diagnose- und kein Befunderhebungsfehler vor, wenn bei einer zutreffenden Diagnose noch weitere Befunde hätten erhoben werden müssen (BGH, Urt. v. 21.12.2010, Az.: VI ZR 284/09)

Maßgabe bei der Bewertung jeglichen ärztlichen Handelns ist nach ständiger Rechtsprechung des BGH der sog. Facharztstandard (BGH, Urt. v. 21.11. 1995 , Az.: VI ZR 341/94). Der Facharztstandard ist ein Sorgfaltsmaßstab zur Beurteilung des ärztlichen Verschuldens. Er beschreibt den Standard, der auf dem Fachgebiet dem gesicherten Stand der medizinischen Wissenschaft entspricht. Dabei muss eine Universitätsklinik den medizinischen Standard in besonderer Weise erfüllen (BGH, Urt. v. 22.09.1987, Az.: VI ZR 238/86)

Nach den Vorschriften zum Behandlungsvertrag wird gemäß § 630a Abs. 2 BGB der Facharztstandard geschuldet. Soweit ein Behandelnder für die von ihm vorgenommene Behandlung nicht befähigt war, wird zudem nach gemäß $\S 630$ h Abs. 4
BGB vermutet, dass die mangelnde Befähigung für den Eintritt der Verletzung des Lebens, des Körpers oder der Gesundheit ursächlich war; d. h. es findet eine Beweislastumkehr zulasten des Arztes statt. Angesichts der Tatsache, dass der Probandenvertrag nicht explizit gesetzlich geregelt ist, jedoch eine vergleichbare Interessenlage wie beim Behandlungsvertrag vorherrscht, dürften, wie oben bereits erwähnt, diese Grundsätze auf die vorliegende Situation analog anwendbar sein.

Zudem ist hinsichtlich der Aufklärung der Probanden festzuhalten, dass die Anforderungen an die Aufklärung umso höher sind, je weniger eine ärztliche Maßnahme medizinisch geboten ist.

\section{Zufallsbefunde}

Zur Beantwortung der oben gestellten Frage bzgl. der Beachtung von Zufallsbefunden ist die dazu ergangene Rechtsprechung heranzuziehen. Hier geht es um die Frage, ob ein ärztlicher Behandlungsfehler vorliegt, wenn ein - auf einem Untersuchungsbild erkennbarer - Befund vorliegt, die Auswertung der entsprechenden Bildgebung jedoch medizinisch nicht geboten war.

Der BGH nimmt hinsichtlich dieser Frage das Berufsbild des Arztes in den Blick. Der BGH betont, dass „das Wohl des Patienten oberstes Gebot und Richtschnur jeden ärztlichen Handelns ist" (BGH, Urt. v. 21.12.2010, Az.: VI ZR 284/09; mit Verweis auf BGH, Urt. v. 26.01.1999, Az.: VIZR 376/97). Ausdrücklich wird verlangt, dass der Arzt auch die Ergebnisse solcher Untersuchungen, die medizinisch nicht verlangt waren mit berufsspezifischer Sorgfalt behandeln muss

„Auf diese Weise gewonnene Erkenntnisse dürfen (...) vom Arzt nicht deshalb ignoriert werden, weil keine Verpflichtung zur Durchführung der entsprechenden Untersuchung bestand." (BGH, Urt. v. 21.12.2010, Az.: VI ZR 284/09).

In diesem Fall kommt eine Garantenhaftung des Arztes infrage, der eine gebotene Kontrolluntersuchung unterlässt (OLG Düsseldorf, Urt. v. 31.01.1991, Az.: 8 U 119/88). Der BGH sieht dabei die Verantwortung des Arztes nicht nur innerhalb seines eigenen Fachgebiets für gegeben an (BGH, Urt. v. 21.12.2010, Az.: ZR 284/09). Der für die Auswertung eines Befunds im konkreten Fall medizinisch verantwortli- che Arzt hat „all die Auffälligkeiten zur Kenntnis und zum Anlass für die gebotenen Maßnahmen zu nehmen, die er aus berufsfachlicher Sicht seines Fachbereichs unter Berücksichtigung der in seinem Fachbereich vorausgesetzten Kenntnisse und Fähigkeiten sowie der Behandlungssituation feststellen muss. Vor in diesem Sinne für ihn erkennbaren ,Zufallsbefunden ' darf er nicht die Augen verschließen.“ (BGH a. a. O.)

Insoweit ist auch auf eine Entscheidung des OLG Hamm vom 12.08.2013 (Az.: 3 U 57/13) hinzuweisen, bei der ein Gynäkologe verurteilt wurde, der einer vorbelasteten Patientin nicht zur Teilnahme am staatlichen Mammografiescreening-Programm geraten hatte (vgl. hierzu auch Wigge / Frigger, Fortschr Röntgenstr 2013, S. 1108 ff.).

\section{Anwendung auf die Situation in der} radiologischen Klinik

Diese von der Rechtsprechung entwickelten Grundsätze sind auf die Situation anzuwenden, dass das MRT in der radiologischen Klinik einer Universitätsklinik oder Krankenhauses nicht zu Untersuchungszwecken im Sinne der medizinischen Behandlung, sondern zu klinischen und/oder wissenschaftlichen Untersuchungen genutzt wird:

Bei der ausschließlich klinisch indizierten Bildgebung liegt danach eine Haftung des verantwortlichen Arztes bzw. der Klinik sowohl nach vertraglichen als auch nach deliktischen Grundsätzen vor. Sofern der Radiologe in diesem Fall (mit-)verantwortlicher Arzt ist, haftet dieser auch unmittelbar.

Problematisch ist die Frage der Haftung bei der Bildgebung bei kombinierter klinischer und wissenschaftlicher Fragestellung. In diesem Fall liegt jedoch (auch) die Behandlung eines Patienten und somit eine genuin ärztliche Tätigkeit vor. Aus diesem Grund müssen die oben genannten Grundsätze in jedem Fall Anwendung finden. Das bedeutet, dass der für die Studie verantwortliche Arzt eine Abklärung durch einen Radiologen veranlassen muss. So muss auch vorgegangen werden, wenn es sich um eine ausschließlich wissenschaftlich veranlasste Bildgebung handelt. Die vom BGH herangezogenen Berufspflichten gelten auch in diesem Fall. 
Die Abklärung sollte angesichts der Tatsache, dass - nach Studien - bei bis zu 3\% aller Probanden eine - meist schwerwiegende bzw. lebensbedrohliche - Anomalie vorliegt, auch regelhaft erfolgen. Daher ergibt sich gleichsam eine Berufspflicht, eine entsprechende Abklärung vorzunehmen.

\section{Studien unter der Leitung von Nichtärzten}

Die ärztlichen Berufspflichten, auf die der BGH rekurriert, begegnen dann Schwierigkeiten, wenn der verantwortliche Studienleiter kein Arzt ist, sondern Psychologe, Naturwissenschaftler oder Mediziner ohne Approbation die Studienleitung innehaben.

Diese Konstellation ist nicht unproblematisch. Dazu führt die Bundesärztekammer in den Leitlinien zur Qualitätssicherung der Magnetresonanztomografie aus:

„Die Magnetresonanztherapie stellt derzeit das modernste aber zugleich technisch aufwendigste Schnittbildverfahren in der Radiologie dar. Die Qualitätssicherung in der MRT hat eine besondere Bedeutung, da wegen der Vielzahl veränderbarer und voneinander abhängiger Messparameter die Fehlermöglichkeiten durch Artefakte und inadäquate Durchführung der Untersuchung erheblich größer sind als bei allen anderen bildgebenden Verfahren. Deshalb kommt nicht nur der technischen Qualitätssicherung, sondern vor allem auch der ärztlichen Qualifikation bei der Indikationsstellung, der Durchführung, der Auswertung und Beurteilung der MRT eine besondere Rolle zu.

Adäquate Bildqualität und diagnostisch verwertbare Ergebnisse einer Magnet-Resonanz-Tomografie (MRT) erfordern eine exakte ärztliche Indikationsstellung, eine zielorientierte und fachkundige Untersuchungstechnik, eine adäquate Darstellung der diagnostisch wichtigen Bildinformationen, eine fachkundige Auswertung der Messdaten sowie eine sorgfältige und nachvollziehbare Dokumentation der Ergebnisse. Da sich die Methode in einer schnellen Weiterentwicklung befindet, ist eine ständige Fortbildung der fachkundigen Ärzte erforderlich. Wegen des raschen Wandels der technischen Möglichkeiten sollen diese Leitlinien nach Bedarf geändert werden.“ (vgl. DÄBl 2000, S. A 2557).
Diese Ausführungen der Bundesärztekammer stützen auf der einen Seite die bereits ausgeführte haftungsrechtliche Argumentation. Insbesondere wird aber deutlich, dass auch die entsprechenden Studien überhaupt nur Sinn machen, wenn Radiologen eingebunden sind, da nur in diesem Fall die entsprechende Qualität der Maßnahme überhaupt notwendig ist. Gerade die Anforderungen an eine ständige Fortbildung der fachkundigen Ärzte kann in diesem Ausmaß nur von Fachärzten der Radiologie sichergestellt werden.

Die Strahlenschutzkommission, die das Bundesministerium für Umwelt, Naturschutz, Bau und Reaktorsicherheit berät, führt in ihren Empfehlungen zur Vermeidung gesundheitlicher Risiken bei Anwendung magnetischer Resonanzverfahren in der medizinischen Diagnostik im Ziffer 8.1.1 aus, dass eine MRT-Untersuchung am Menschen „nur eine Person anordnen darf, die zur Ausübung des ärztlichen Berufs berechtigt ist und eine besondere Qualifikation auf dem Gebiet der NMR-Untersuchung nachweisen kann.“

Nach Ziffer 8.1.2 muss bei der Anwendung am Menschen ständig ein sachkundiger Arzt anwesend sein. Ebenso darf die MRTAnlage nur von Personen betrieben werden, die die notwendigen Kenntnisse besitzen, um sie bestimmungsgemäß zu betreiben und um auf Havarie- und Notfallsituationen entsprechend reagieren $\mathrm{zu}$ können. Den Leitlinien der Bundesärztekammer sowie den Empfehlungen der Strahlenschutzkommission käme in einem Haftungsprozess erhebliche Relevanz zu. Der Facharztstandard muss daher in jedem Fall eingehalten werden.

\section{Expliziter Verzicht des Proban- den / Patienten}

Ein Verzicht des Probanden / Patienten auf die Aufklärung über einen möglichen Befund ist möglich. Dieser muss jedoch umfassend und eindeutig erklärt und zu Dokumentationszwecken zu seinen Unterlagen genommen werden. Um sicherzustellen, dass der entsprechende Verzicht des Probanden / Patienten wirksam ist, sollte im Rahmen seiner Aufklärung über das Forschungsvorhaben über die Häufigkeit von Zufallsbefunden und über die Schwere der möglichen Befunde aufgeklärt werden. Der Patient muss wissen, worum es geht. Ansonsten drohen bei Versagung der Aufklärung auch erhebliche strafrechtli- che Konsequenzen. Die sonstige Feststellung einer Unzumutbarkeit der Aufklärung unterliegt strengen Anforderungen, da die Selbstbestimmung des Patienten gewahrt bleiben muss (siehe hierzu: Ulsenheimer, Arztstrafrecht in der Praxis, 3. Auflage, Rn. 63).

\section{Haftung der Radiologen und der Klinik}

Eine Haftung der Ärzte und / oder der Klinik könnte sich aus 2 Gründen ergeben:

Zum einen könnten die Ärzte oder auch die Klinik selbst Vertragspartner der Probanden/Patienten sein. In diesem Fall haften die Ärzte und / oder die Klinik auch für das Tun und Unterlassen ihrer sogenannten Erfüllungsgehilfen im Rahmen des Behandlungsvertrages gemäß $§ 278$ BGB.

Eine Haftung des Chefarztes oder Direktor der radiologischen Klinik könnte sich aus $\S 831$ BGB ergeben. Dort ist die Haftung für einen Verrichtungsgehilfen geregelt. Diese Vorschrift bestimmt:

„Wer einen anderen zu einer Verrichtung bestellt, ist zum Ersatz des Schadens verpflichtet, den der andere in Ausführung der Verrichtung einem Dritten widerrechtlich zufügt. Die Ersatzpflicht tritt nicht ein, wenn der Geschäftsherr bei der Auswahl der bestellten Person und, sofern er Vorrichtungen oder Gerätschaften zu beschaffen oder die Ausführung der Verrichtung zu leiten hat, bei der Beschaffung oder der Leitung die im Verkehr erforderliche Sorgfalt beobachtet oder wenn der Schaden auch bei Anwendung dieser Sorgfalt entstanden sein würde.“

Im Wesentlichen wird durch die Haftung nach $\S 831$ BGB ein sog. Organisationsverschulden erfasst. Dies wäre dann der Fall, wenn der Direktor der radiologischen Klinik nicht dafür sorgen würde, dass z. B. ein fachlich qualifizierter Arzt die MRT-Untersuchung betreut oder die entsprechende Indikation für die Kontrastmittelgabe erstellt.

\section{Haftung für Kontrastmittel- indikation und -applikation $\nabla$}

Die Stellung der rechtfertigenden Indikation für die Kontrastmittelgabe muss durch den Radiologen erfolgen. Sie ist 
nicht auf nichtärztliches Personal delegierbar.

Die Durchführung der Kontrastmittelapplikation ist dagegen eine ärztliche Tätigkeit, die auf einen medizinisch-technische/r Radiologieassistent/-in (MTRA) mit Fachkenntnissen delegiert werden kann. Nach den oben genannten Haftungsgrundsätzen haften die Ärzte oder ggf. die Klinik für das Fehlverhalten der MTRA nach den Grundsätzen des Verrichtungs- und/oder Erfüllungsgehilfen ( $\S \S$ 278, 831 BGB). Zu beachten ist, dass der Radiologe sich bei intravenöser Kontrastmittelgabe in unmittelbarer Nähe aufhalten muss. Dies gilt insbesondere angesichts der zahlreichen möglichen Nebenwirkungen des Kontrastmittels, insbesondere allergischer Reaktionen (vgl. Stellungnahme BÄK/KBV, Persönliche Leistungserbringung, Möglichkeiten und Grenzen der Delegation ärztlicher Leistungen v. 29.08.2008, DÄBl. 2008 A 2173; vgl. auch Anhang zur Anlage 24 des BMVÄ ,Vereinbarung über die Delegation ärztlicher Leistungen an nichtärztliches Personal in der ambulanten vertragsärztlichen Versorgung gemäß § 28 Abs. 1 S. 3 SGB V“). Die Applikation kann daher von einer MTRA vorgenommen werden, wenn diese von einem Facharzt für Radiologie beaufsichtigt wird. Die Beaufsichtigung der MTRA durch einen Weiterbildungsassistenten ist nur unter bestimmten Voraussetzungen möglich. Weiterbildungsassistenten dürfen insofern selbstständig tätig werden, wie dies ihrem Weiterbildungsstand entspricht (Narr, Ärztliches Berufsrecht, Band 1, S. 274). Ein Weiterbildungsassistent darf daher ,nur zu solchen Aufgaben herangezogen werden, die er aufgrund seiner bisherigen Erfahrungen und Fähigkeiten beherrscht." (Narr, a.a.O.). Die Verantwortung für die Tätigkeit des Weiterbildungsassistenten obliegt dem ausbildenden Arzt.

Im Rahmen gesetzlicher Bestimmungen wird Weiterbildungsassistenten daher nur ein enger Handlungsspielraum zugestanden. Hier ist z. B. auf die Richtlinie zur Ambulanten Spezialfachärztlichen Versorgung (ASV-RL) des G-BA vom 21.03.2013 (BAnz AT 19.07.2013 B1) hinzuweisen. § 3 Abs. 4 S. 4 der Richtlinie verlangt, dass Ärzte in Weiterbildung entsprechend dem Stand ihrer Weiterbildung unter Verantwortung eines zur Weiterbildung befugten Mitglieds des interdisziplinären Teams eingebezogen werden, wobei sie die Diagnosestellung und leitende
Therapieentscheidungen nicht erbringen dürfen. Auch ein ermächtigter Krankenhausarzt darf sich nach $\S 32$ a Ärzte-ZV nicht von Weiterbildungsassistenten vertreten lassen, was auch im Falle von Radiologieleistungen gilt (Quaas / Zuck, Medizinrecht, § 16, Rn. 78). Haftungsrechtlich ist auch hier grundsätzlich der Facharztstandard gefordert. Auch ist der aus dem Aufklärungsrecht bekannte Grundsatz zu beachten, dass die Anforderungen umso höher sind, je weniger ein Eingriff medizinisch geboten ist. Daher ist bei dem Einsatz von Weiterbildungsassistenten Vorsicht geboten.

\section{Erstellung zusätzliche Bilder $\nabla$}

Aufgrund der oben genannten Sorgfaltspflichtanforderungen und berufsethischen Verpflichtungen müssen u.E. entweder zusätzliche Bilder angefertigt werden, um einen korrekten Befund zu ermöglichen, falls sich aufgrund der nichtmedizinischen MRT-Untersuchungen Anhaltspunkte für das Vorliegen einer Erkrankung ergeben, die jedoch mit letzter Sicherheit nur durch einen zusätzlichen Befund ausgeschlossen oder bestätigt werden kann. Alternativ müssen weitere MRT-Untersuchungen durch einen Radiologen durchgeführt werden.

\section{Zusätzliche Befundung von Bil- dern \\ $\nabla$}

Wie oben dargelegt, besteht eine Verpflichtung des Wissenschaftlers, die Bilder zur Abklärung an den Radiologen weiterzugeben.

\section{Anwesenheit eines Radiologen $\nabla$}

Im Rahmen der medizinischen Patientenversorgung gilt für die Durchführung von MRT-Untersuchungen folgendes:

MRT-Untersuchungen kann nur ein Arzt anordnen und befunden. Die technische Durchführung von MRT-Untersuchungen kann er an nichtärztliche Mitarbeiter delegieren. In diesem Fall muss er mit den die Untersuchung durchführenden nichtärztlichen Mitarbeitern in der Weise in Verbindung stehen, dass er die entstehenden Aufnahmen bewerten und den weiteren Gang der Untersuchung steuern kann. Bei Risikopatienten muss sich der Arzt in unmittelbarer Nähe aufhalten; ausrei- chend ist auch die unmittelbare Nähe eines anderen Arztes, der das mit der Leistung verbundene Risiko beherrscht.

Teilweise ist von Krankenhäusern in Nutzungsordnungen für die MRT-Systeme vorgeschrieben, dass ein Arzt die theoretische und praktische Expertise im Bereich des MRT-Gerätes vorweisen muss. Diese Vorgaben stützen sich auf die Empfehlungen der Strahlenschutzkommission zur Sicheren Anwendung magnetischer Resonanzverfahren in der medizinischen Diagnostik, die ebenfalls die Durchführung durch sachkundiges Personal und sogar den ständigen optischen und akustischen Kontakt zwischen Untersucher und Untersuchungsperson verlangen. Diese in den Nutzungsordnungen formulierten Vorgaben dürften dem Stand der medizinischen Wissenschaft entsprechen. Dies gilt insbesondere für Universitätskliniken, die haftungsrechtlich im Regelfall schärfer beurteilt werden als Allgemeinkrankenhäuser (s. o.). Sollten die personellen Anforderungen nicht eingehalten werden, so ist davon auszugehen, dass die die MRTUntersuchung unter Verstoß gegen die Anforderungen an den Behandlungsvertrag nach $\S \S 630 a$ ff. BGB erfolgt, was nach den oben genannten Grundsätzen haftungsauslösend wirken kann.

In diesem Zusammenhang ist auch auf das Gesetz zum Schutz vor nichtionisierender Strahlung bei der Anwendung am Menschen (NiSG) hinzuweisen. Danach dürfen in Ausübung der Heil- oder Zahnheilkunde am Menschen beim Betrieb von Anlagen, die nichtionisierende Strahlung aussenden können, die in einer Rechtsverordnung nach $\S 2$ Abs. 1 NiSG für bestimmte Anwendungsarten festgelegten Werte nur dann überschritten werden, wenn eine berechtigte Person hierfür die rechtfertigende Indikation gestellt hat. Die Anforderungen nach $\S 2$ Abs. 2 NiSG verlangen dazu neben einer Approbation eine entsprechende Fachkunde, die allerdings bisher nicht näher definiert ist.

Die entsprechende Rechtsverordnung ist zwar bisher nicht ergangen. Vorsorglich sollte jedoch, bis Grenzwerte festgelegt sind, bei jeder Untersuchung ein Arzt mit der entsprechenden Fachkunde anwesend sein. Nach dem Gesetzeswortlaut muss dies nicht zwingend ein Radiologe sein, sondern könnte z.B. auch durch einen Inhaber mit der Zusatzweiterbildung „Magnetresonanztomografie - fachgebunden“ erfolgen. 
Nach $\S 2$ Abs. 4 der Weiterbildungsordnungen der Landesärztekammern beinhaltet eine Zusatzweiterbildung die Spezialisierung in Weiterbildungsinhalten, die zusätzlich zu den Facharzt- und Schwerpunktweiterbildungsinhalten abzuleisten sind, sofern nichts anderes in Abschnitt $C$ geregelt ist. Der Erwerb der Zusatzbezeichnung setzt die Ableistung der vorgeschriebenen Weiterbildungszeiten und Weiterbildungsinhalte voraus (24 Monate bei einem Weiterbildungsbefugten für Radiologie gemäß $\S 5$ Absatz $1 \mathrm{WO}$, davon können bis zu 12 Monate bei einem Weiterbildungsbefugten gemäß $\S 5 \mathrm{Ab}$ satz 1 Satz 2 WO und 12 Monate im ambulanten Bereich abgeleistet werden).

Angesichts der Vielzahl von möglichen schwerwiegenden Krankheitsbildern, die sich aufgrund von Zufallsbefunden auch im Rahmen einer zu wissenschaftlichen Zwecken ohne medizinische Indikation durchgeführte MRT-Untersuchung ergeben können, ist die Anwesenheit und Mitwirkung eines Facharztes für Radiologie geboten. Dies ergibt sich daraus, dass auf diese Weise eine Haftung aufgrund von nicht weiter verfolgten Zufallsbefunden mit größerer Wahrscheinlichkeit vermieden werden kann. Insbesondere gilt dies dann, wenn sich das MRT-Gerät innerhalb einer radiologischen Abteilung oder eines radiologischen Instituts befindet.

Es ist zudem darauf hinzuweisen, dass nach den Empfehlungen zur sicheren Anwendung magnetischer Resonanzverfahren in der medizinischen Diagnostik der Strahlenschutzkommission des Bundesministeriums für Umwelt, Naturschutz und Reaktorsicherheit eine solche Überwachung sichergestellt sein muss, damit die durch bekannte Wirkungsmechanismen eintretenden Schäden vermieden werden (Ziffer 8.2 der Empfehlungen).

Nach den Empfehlungen der Strahlenschutzkommission muss die Durchführung der Untersuchung

„unter der Verantwortung eines mit den spezifischen gesundheitsrelevanten Gegebenheiten von MR-Untersuchungen vertrauten fachkundigen Arztes erfolgen. Insbesondere muss die adäquate Versorgung bei speziellen Gesundheitsrisiken der untersuchten Personen sichergestellt sein. (...) Eine sich aus der Untersuchung ergebende oder auch im Rahmen allgemeiner Gesundheitsrisiken auftretende angemessene ärztliche Versorgung ist si- cherzustellen.“ (Ziffer 8.2 der Empfehlungen)

Die entsprechende Überwachungs- und Versorgungsleistung, die hier vorgegeben ist und damit auch mit großer Wahrscheinlichkeit für die Bestimmung des Facharztstandards im Rahmen dieser Untersuchungen herangezogen würde, kann nur durch die ständige Anwesenheit eines Facharztes für Radiologie erfüllt werden.

\section{Aufklärungspflicht bzgl. Einsatz von Kontrastmitteln und der MRT- Untersuchung}

Bei der Einbringung des Kontrastmittels ist von einer Aufklärungspflicht des Arztes bzgl. etwaiger Risiken und Nebenwirkungen auszugehen.

\section{Aufklärungspflicht}

Eine solche Aufklärungspflicht könnte sich aus 2 Gründen ergeben: Zum einen ist hier an den Bereich der Risikoaufklärung zu denken. Das bedeutet, dass der Patient bevor er sich zu einer Einwilligung in eine Behandlung entschließt, die notwendigen Informationen über das Für und Wider erhält (vgl. BGH, Urt. v. 14.03.2006, Az.: VI ZR 279/06). Dabei muss auf Gefahren und Folgeschäden hingewiesen werden, die trotz Anwendung der ärztlichen Sorgfalt nicht ausgeschlossen werden könnten. Im Rahmen der Kontrastmittelgabe ist dabei insbesondere an zahlreiche Nebenwirkungen zu denken. Auch diese sind verpflichtender Teil der ärztlichen Aufklärung (vgl. BGH, Urt. v. 27.10.1981, Az.: VI ZR 69/80).

Im Falle eines Behandlungsvertrags ist in $\S$ 630e Abs. 1 BGB folgendes geregelt:

„Der Behandelnde ist verpflichtet, den Patienten über sämtliche für die Einwilligung wesentlichen Umstände aufzuklären. Dazu gehören insbesondere Art, Umfang, Durchführung, zu erwartende Folgen und Risiken der Maßnahme sowie ihre Notwendigkeit, Dringlichkeit, Eignung und Erfolgsaussichten im Hinblick auf die Diagnose oder die Therapie. Bei der Aufklärung ist auch auf Alternativen zur Maßnahme hinzuweisen, wenn mehrere medizinisch gleichermaßen indizierte und übliche Methoden zu wesentlich unterschiedlichen Belastungen, Risiken oder Heilungschancen führen können.“
Daraus folgt, dass eine umfassende Risikoaufklärung in jedem Fall durchgeführt werden muss. Dies gilt im Übrigen auch für die Verwendung des MRT an sich. Auch die dadurch erzeugte - zwar nichtionisierende, aber magnetische - Strahlung hat Auswirkungen auf den Körper. Das gilt zum einen für die Magnetfelder an sich, zum anderen aber auch für Hilfsmittel (z.B. Herzschrittmacher) am Körper der Patienten. Auch dadurch ergeben sich aufklärungsbedürftige Risiken für den Eingriff. Nicht umsonst hat der Gesetzgeber das NiSG erlassen.

Die an die Aufklärung zu stellenden Anforderungen können zudem bei wissenschaftlichen Studien ohne klinische Indikation noch einmal erhöht sein. Eine Erhöhung der Aufklärungspflichten liegt einerseits dann vor, wenn ein ärztlicher Eingriff medizinisch nicht geboten ist. Dann muss der Patient umso ausführlicher und eindrücklicher informiert werden. Diese Notwendigkeit einer umfangreicheren Aufklärungspflicht besteht vor allem bei plastischen Schönheitsoperationen (BGH, Urt. v. 06.11.1990, Az.: VIZR 8/90). Gleichwohl ergibt sich eine Vergleichbarkeit mit der Situation bei wissenschaftlichen Studien, da dort - zumindest ex-ante - eine medizinische Gebotenheit nicht gegeben ist. Daher besteht gerade in diesen Fällen eine besondere Pflicht zur umfassenden Aufklärung.

Darüber hinaus besteht eine besondere Aufklärungspflicht für den Arzt bei Eingriffen zur Diagnosestellung. Darunter versteht man diagnostische Eingriffe ohne therapeutischen Eigenwert. Dies gilt nur dann nicht, wenn „dieser Eingriff gerade dringend oder sogar vital indiziert ist; denn andernfalls dient er nicht der Abwendung einer akuten Gefahr für Leben und Gesundheit. Im letzteren Fall, welcher der hier gegebenen Situation entspricht, sind dem Patienten selbst entfernt liegende Komplikationsmöglichkeiten in angemessener Weise darzutun“ (OLG Hamm, Urt. v. 19.12.1979, 13 U 268/78).

Auch diese Situation weist starke Ähnlichkeiten zu der Situation bei einer wissenschaftlichen Studie auf. Daher dürften die Rechtsgrundsätze übertragbar sein. Zudem sollte bei der Aufklärung erwähnt werden, dass die Forschungsmaßnahme dem Prüfplan der entsprechenden Studie folgt und das Vorgehen von demjenigen im Bereich der Krankenversorgung abwei- 
chen kann (Pramann / Albrecht, Forschung im Krankenhaus, S. 25 f.).

\section{Aufklärungspflichtige Person}

Die Aufklärung ist eine ärztliche Aufgabe, wobei keine Identität zwischen dem durchführenden und dem aufklärenden Arzt verlangt wird. Er übernimmt eine Garantenstellung gegenüber dem Aufzuklärenden, aus der er auch in die - zivil und strafrechtliche - Haftung genommen werden kann (BGH, Urt. v. 22.04.1980, Az.: VI ZR 37/79). Nach den Vorschriften über den Behandlungsvertrag gemäß $\S 630 \mathrm{e}$ Abs. 2 Nr. 1 BGB muss die Aufklärung mündlich durch den Behandelnden oder durch eine Person erfolgen, die über die zur Durchführung der Maßnahme notwendige Ausbildung verfügt.

Angesichts der besonders hohen Anforderungen an die Aufklärung aufgrund der nichtzwingenden Indikation sollte diese Aufklärung nur durch einen Facharzt für Radiologie, der mit MRT vertraut ist durchgeführt werden. Die Aufklärung durch einen Arzt mit der fachgebundenen Schwerpunktbezeichnung wäre denkbar, jedoch muss dieser ebenfalls das geforderte Aufklärungsniveau erreichen. Grundsätzlich ist jeder Arzt für die Aufklärung innerhalb seines Fachgebiets verantwortlich.
Pflichten des Arztes hinsichtlich der Diagnoseaufklärung

In der Rechtsprechung wird auch eine Pflicht des Arztes angenommen, Patienten einen Befund mitzuteilen. Rechtsdogmatisch wird diese allerdings als Nebenpflicht aus dem Behandlungsvertrag zwischen Arzt bzw. Krankenhaus und Patient hergeleitet. Dies kann man, sofern der Patient nicht verzichtet hat (s.o.), auf den Probandenvertrag übertragen. Dies bedeutet, dass eine solche Pflicht zur Diagnoseaufklärung besteht und sich daraus ggf. auch entsprechende strafrechtliche Konsequenzen ergeben können (Ulsenheimer, Arztstrafrecht in der Praxis, 3. Auflage, Rn. 63). Dies gilt insbesondere dann, wenn davon wichtige Entscheidungen für das weitere Vorgehen des Patienten vorgehen (Geiß/Greiner, Arzthaftpflichtrecht, 5. Auflage, S. 238). Ansonsten besteht eine Mitteilungspflicht jedoch nur im Rahmen der Behandlungs- und Risikoaufklärung (Geiß/Greiner, a.a.O.). Allerdings kann sie im Fall der Diagnoseaufklärung auch kontraindiziert sein (Deutsch/Spickhoff, Medizinrecht, Rn. 267; Pramann/Albrecht, Forschung im Krankenhaus S. 22)

\section{Ergebnis}

Die MRT-Anwendung zu Forschungszwecken ist bisher nur teilweise und lückenhaft geregelt. Dies bedeutet jedoch nicht, dass sie schrankenlos möglich ist. Vielmehr sind die schon bestehenden rechtlichen Regelungen, verbunden mit Äußerungen der Rechtsprechung, heranzuziehen. Probleme ergeben sich dabei insbesondere im Haftungsrecht sowie bei Studien unter der Leitung von Nichtärzten. Insbesondere bei letzterer Problematik ist der Gesetzgeber gefordert. In den einzelnen Einrichtungen sollten zudem Nutzungsordnungen ausgearbeitet werden, die die oben genannten rechtlichen Maßstäbe erfüllen.

\section{Prof. Dr. Peter Wigge}

Rechtsanwalt, Fachanwalt für Medizinrecht

\section{Lic. iur. can. Urs Fabian Frigger}

Rechtsanwalt

Rechtsanwälte Wigge

Scharnhorststr. 40

48151 Münster

Telefon: (0251) 53595-0

Telefax: (0251) 53595-99

E-Mail: kanzlei@ra-wigge.de

www.ra-wigge.de 